\title{
Percepción de los Estudiantes de Medicina Veterinaria acerca de los Perros vagabundos y sus Estrategias de Control en Lima, Perú
}

\author{
Perception of Veterinary Medicine Students on Stray Dogs and their Control \\ Strategies in Lima, Peru
}

Micaela Vizquerra B. ${ }^{1,4}$, Felipe San Martín H. ${ }^{2}$, Iván Carbajal G. ${ }^{\text {, }}$, Néstor Falcón P. ${ }^{1}$

\section{Resumen}

\begin{abstract}
El objetivo del estudio fue determinar la percepción de los estudiantes de la carrera de medicina veterinaria (MV) acerca de la presencia de perros vagabundos y sus estrategias de control en Lima, Perú. Se elaboró un instrumento de auto-aplicación con preguntas de alternativas múltiples, de una o más opciones de respuesta y se aplicó en forma anónima a los estudiantes de MV de dos universidades de Lima. Se hizo una validación previa del contenido por jueces expertos (prueba de distribución binomial) y prueba piloto en estudiantes de MV de una tercera universidad, para evaluar la consistencia interna (prueba estadística alfa de Cronbach). Se analizaron 457 encuestas. El $36.5 \%$ de los encuestados perciben que los perros vagabundos en las calles tenían dueño. Los encuestados consideraron que los principales problemas que sufren estos animales eran desnutrición $(86.7 \%)$, parasitosis $(62.8 \%)$ y accidentes $(54.9 \%)$, y los principales problemas que ocasionaban eran la reproducción no deseada $(76.1 \%)$, zoonosis (47.9\%) y ruptura de bolsas de basura (40.7\%). Los estudiantes le atribuyen la responsabilidad de enfrentar el problema a la municipalidad del distrito (86.4\%) y el Ministerio de Salud (60.6\%). Para controlar el problema consideran las esterilizaciones (79.0\%), adopciones $(70.9 \%)$ y castraciones $(60.8 \%)$ como las opciones más viables.
\end{abstract}

Palabras clave: perros vagabundos; parasitosis; zoonosis; esterilizaciones; castraciones

\footnotetext{
${ }^{1}$ Grupo de Salud Pública Veterinaria (SAPUVET-PERÚ), Facultad de Medicina Veterinaria y Zootecnia, Universidad Peruana Cayetano Heredia, Lima, Perú

${ }^{2}$ Facultad de Medicina Veterinaria, Universidad Nacional Mayor de San Marcos, Lima, Perú

${ }^{3}$ Asociación de Egresados de Nutrición, Facultad de Medicina, Universidad Nacional Mayor de San Marcos, Lima, Perú

${ }^{4}$ E-mail: micaela.vizquerra@upch.pe
}

Recibido: 3 de febrero de 2017

Aceptado para publicación: 10 de junio de 2017 
The aim of this study was to determine the perception of the veterinary medicine (VM) students about the presence of stray dogs and their control strategies in Lima. Peru. For this purpose, a self-application questionnaire was designed with multiplechoice questions with one or more response options. It was anonymously applied to VM students from two universities in Lima, after validation of content by expert judges (Binomial distribution test) and a pilot test to students of VM from a third university to assess the internal consistency (Cronbach's alpha statistical test). A total of 457 surveys were analyzed. Results showed that $36.5 \%$ (167) of the respondents perceived stray dogs on the streets had owner. Respondents felt that the main problems felt by these animals were malnutrition (86.7\%) parasitism (62.8\%) and accidents (54.9\%), and the main problems stray dogs cause were unwanted reproduction $(76.1 \%)$, zoonoses $(47.9 \%)$ and breaking of garbage bags $(40.7 \%)$. Students attribute responsibility to address the problem to district municipalities (86.4\%) and the Ministry of Health (60.6\%). To control the problem, students considered sterilizations (79.0\%), adoptions (70.9\%) and neutering $(60.8 \%)$ as the most viable options.

Key words: stray dogs; parasitism; zoonosis; sterilization; neutering

\section{INTRODUCCIÓN}

El crecimiento descontrolado de la población canina se ha convertido en un problema para las autoridades de salud en muchas ciudades de países en desarrollo. Por ejemplo, una estimación realizada en el distrito de San Martín de Porres, Lima (Perú), muestra un crecimiento de $16 \%$ en los seis últimos años (Arauco et al., 2014).

Como perro vagabundo o callejero se define a todo aquel que, aun teniendo dueño, transita libremente por las áreas públicas sin control directo (OIE, 2010). Estos animales buscan alimento entre la basura y desperdicios de las calles y mercados y, ante la incapacidad de obtener un hogar, utilizan las estructuras urbanas abiertas y abandonadas como refugio temporal (Molina et al., 2006), exponiéndose a violencia y accidentes. En ellos se suele observar lesiones en la piel, cojeras, desnutrición y, consecuentemente, baja condición corporal; además de representar un reservorio de enfermedades que afec- tan su salud y que pueden ser de carácter zoonótico (Ochoa et al., 2014).

Los perros vagabundos pueden ocasionar diversos problemas de salud pública; entre ellos, accidentes por mordedura (Schvartzman y Pacín, 2005), contaminación por heces y transmisión de enfermedades zoonóticas (Ochoa et al., 2014). En el Perú, el costo por atención hospitalaria debido a mordeduras de perros en un hospital de niños se ha calculado en US\$ 210 por paciente (Romero et al., 2013).

En cuanto a la contaminación ambiental por heces de perros, se debe de tener en cuenta que un perro de raza grande puede excretar, en promedio, $340 \mathrm{~g} /$ día de heces (Salamanca et al., 2011) y, a través de ello, diseminar huevos de diversos parásitos (Chávez et al., 2000; La Rosa et al., 2001; López et al., 2005; Ramírez et al., 2014). Entre las zoonosis más importantes que pueden transmitir los perros vagabundos se encuentran la toxocariosis, giardiasis, rabia y leptospirosis. Por ejemplo, se ha reportado 
47\% de prevalencia de Toxocara canis en niños en San Juan de Lurigancho (Breña et al., 2011).

Entre las estrategias de control de población de perros vagabundos se pueden mencionar las de corto plazo, como la esterilización, la eliminación (eutanasia), la adopción y los albergues. Entre las estrategias de largo plazo se tiene la tenencia responsable. Sin embargo, para tener éxito en estas actividades se requiere conocer la percepción de los diversos actores acerca de la presencia de perros vagabundos, su impacto y las medidas de control que, desde cada una de sus percepciones, están dispuestos a poner en práctica. Los estudiantes de medicina veterinaria son parte de la población que debe de abordar este problema, dado que como profesionales lo tendrán que afrontar; de allí que el objetivo del estudio fue conocer la percepción de estos estudiantes acerca de la presencia de perros vagabundos y de las estrategias de su control.

\section{Materiales y Métodos}

El estudio de investigación transversal tipo prevalencia se desarrolló en las facultades de medicina veterinaria de dos universidades ubicadas en Lima Metropolitana, Perú. El estudio contó con la aprobación del Comité de Ética Institucional de la Universidad Peruana Cayetano Heredia (Constancia 37618-15).

La población objetivo estuvo constituida por la totalidad de estudiantes matriculados en ambas facultades en el periodo académico 2015-I. El tamaño de muestra se estableció mediante la fórmula de tamaño de muestra para estimar una proporción, con ajuste por población finita ( $\mathrm{N}=630$, estudiantes matriculados). Las restricciones fueron $\mathrm{p}=0.50$ (utilizado cuando no se conoce un valor referencial), nivel de confianza del $95 \%$, error del 5\% y una tasa de respuesta de $85 \%$ (Seoane et al., 2007). El tamaño de muestra mínimo calculado fue de 281 encuestas, las que se dividieron proporcionalmente al tamaño de la población de estudiantes matriculados en cada institución (125 y 156 encuestas para la universidad 1 y 2 , respectivamente).

El instrumento consistió en un cuestionario estructurado para auto-aplicación, de preguntas con alternativas múltiples, de una o más opciones de respuesta por pregunta, pre-codificadas (Sánchez, 2007). Se dividió en dos secciones: datos generales (universidad de procedencia, edad, género, zona de residencia y año de estudios) y preguntas específicas (percepción de tenencia de propietarios, de problemas que sufren y ocasionan los perros vagabundos, del responsable del control del problema de perros vagabundos, del estudiante acerca de las acciones de control para el problema y declaración de haber llevado un curso relacionado a la tenencia responsable de animales de compañía).

Dichos ítems fueron sometidos a una prueba de validación de contenido por jueces expertos, empleando la prueba de distribución binomial. Posteriormente se realizó una prueba piloto en 30 estudiantes de medicina veterinaria de una tercera universidad de Lima, para evaluar la consistencia interna; es decir, determinar la concordancia entre los ítems empleando la prueba estadística alfa de Cronbach (Garrido et al., 2010). Cuando este superó el 0.6 se dio como aceptable el cuestionario.

Para la recolección de muestras o datos se coordinó con las autoridades de cada facultad para explicar los objetivos y procedimientos del estudio. Una vez obtenida la aprobación, se realizó la encuesta en cada aula, durante las horas de clase, realizando las indicaciones para la autoadministración del cuestionario, y absolviendo las dudas que se presentaron durante su aplicación. El cuestionario fue anónimo y la participación fue voluntaria. 
Cuadro 1. Características demográficas de los estudiantes de la carrera de medicina veterinaria participantes del estudio. Lima, $2015(\mathrm{n}=457)$

\begin{tabular}{llcc}
\hline \multirow{2}{*}{ Variable } & Estrato & \multicolumn{2}{c}{ Estudiantes } \\
\cline { 3 - 4 } Edad & Menos de 18 & $\mathrm{n}$ & $\%$ \\
& $18-20$ & 74 & 16.2 \\
& $21-24$ & 257 & 56.2 \\
& Más de 25 & 84 & 18.4 \\
Sexo & Hombre & 42 & 9.2 \\
& Mujer & 163 & 35.7 \\
Zona de residencia & Centro & 294 & 64.3 \\
(Cono) & Norte & 131 & 28.7 \\
& Este & 120 & 26.3 \\
& Sur & 97 & 21.2 \\
Año de estudios & Callao & 85 & 18.6 \\
& Primero & 24 & 5.3 \\
& Segundo & 137 & 30.0 \\
& Tercero & 117 & 25.6 \\
& Cuarto & 83 & 18.2 \\
& Quinto & 61 & 13.3 \\
& & 59 & 12.9 \\
\hline
\end{tabular}

La información recolectada en los cuestionarios físicos se ingresó a una plantilla de Microsoft Excel 2013 diseñada para tal fin. Posteriormente, la información de la plantilla se exportó al programa SPSS para Windows v. 22, construyéndose el libro de datos y el diccionario de variables correspondiente. Asimismo, la información fue revisada para validar el ingreso de datos y detectar información no coherente.

Las variables cualitativas (género, año de estudios, zona de procedencia) y la variable cuantitativa continua edad (convertida a rangos o intervalos) fueron resumidas en tablas de frecuencia. De la misma forma, las respuestas de la encuesta en la sección de preguntas específicas son presentadas como frecuencia absoluta y relativa.

\section{Resultados}

El estudio recolectó 457 encuestas, de las cuales $51.9 \%$ (237) correspondió a la universidad 1 y $48.1 \%$ (220) a la universidad 2. La mayoría de los entrevistados tenían entre 18 y 20 años de edad, con predominio de estudiantes mujeres. La zona de residencia fue principalmente el Centro, Norte y Este de Lima. La mayoría de los encuestados se agrupaban entre el primer y segundo año de estudio. El detalle se observa en el Cuadro 1. 
Cuadro 2. Percepción de los estudiantes de la carrera de medicina veterinaria acerca de los principales problemas que sufren y ocasionan los perros vagabundos ${ }^{1}$. Lima, 2015 $(\mathrm{n}=457)$

\begin{tabular}{llcc}
\hline \multirow{2}{*}{ Pregunta } & Alternativas & \multicolumn{2}{c}{ Respuestas } \\
\cline { 3 - 4 } & & $\mathrm{n}$ & $\%$ \\
\hline Problemas de salud & Desnutrición & 396 & 86.7 \\
& Violencia física & 224 & 49.0 \\
& Accidentes & 251 & 54.9 \\
& Parasitosis & 287 & 62.8 \\
& Otros & 45 & 9.8 \\
Problemas que & Ensucian las calles con heces y orina & 151 & 33.0 \\
& Rompen bolsas de basura & 186 & 40.7 \\
& Accidentes por mordedura & 101 & 22.1 \\
& Transmiten enfermedades (zoonosis) & 219 & 47.9 \\
& Reproducción descontrolada & 348 & 76.1 \\
& Producen ruidos molestos & 15 & 3.3 \\
\hline
\end{tabular}

${ }^{1}$ Pregunta con opción de respuesta múltiple

En relación a la percepción que tienen los estudiantes sobre los perros vagabundos, se encontró que el 36.5\% (167) afirmaron que los perros vagabundos tenían dueño, que los problemas que sufren estos animales eran la desnutrición, las parasitosis y los accidentes, y que los principales problemas que ocasionan eran la reproducción no deseada, las zoonosis y la ruptura de bolsas de basura (Cuadro 2).

Los encuestados tienen la percepción de que nadie, que los grupos proteccionistas o que los albergues son los que enfrentan el problema de los perros vagabundos. En contraste, cuando se preguntó quién debería enfrentar este problema, los encuestados dieron la responsabilidad a las municipalidades distritales y al Ministerio de Salud. En cuanto a la solución, se mencionan las esterilizaciones de hembras, las adopciones y la castración de machos como las opciones más viables (Cuadro 3). Por último, el 74\% (338) mencionó haber abordado el tema de tenencia responsable durante la carrera.

\section{Discusión}

Las personas encuestadas fueron jóvenes, con mayor porcentaje de estudiantes de los primeros años, debido principalmente a la dificultad de encuestar a los de años superiores, ya que se encontraban fuera de los campus universitarios atendiendo sus prácticas pre-profesionales. La mayor proporción de mujeres encuestadas obedece a la mayor proporción de mujeres que estudian la carrera de Medicina Veterinaria en la actualidad (Paredes y Zuazo et al., 2014).

El estudio muestra una baja percepción de los encuestados acerca de la propiedad de perros que suelen encontrarse en la calle. Es posible que se deba al desconocimiento de la definición de perro vagabundo expresa- 
Cuadro 3. Percepción de los estudiantes de la carrera de medicina veterinaria acerca de las entidades responsables y la mejor solución para eliminar el problema de los perros vagabundos. Lima, $2015(\mathrm{n}=457)$

\begin{tabular}{llcc}
\hline \multirow{2}{*}{ Pregunta } & Alternativas & \multicolumn{2}{c}{ Respuestas } \\
\cline { 2 - 4 } ¿Quién está enfrentando el & Los vecinos o la comunidad & $\mathrm{n}$ & $\%$ \\
problema de los perros & Albergues & 43 & 9.4 \\
vagabundos? & Grupos proteccionistas o animalistas & 185 & 32.8 \\
& La municipalidad del distrito & 47 & 10.5 \\
& Nadie lo enfrenta & 192 & 42.0 \\
¿Quién debería enfrentar el & Los vecinos o la comunidad & 91 & 19.9 \\
problema de los perros & Albergues & 55 & 12.0 \\
vagabundos? & Grupos proteccionistas o animalistas & 75 & 16.4 \\
& La municipalidad del distrito & 395 & 86.4 \\
& El Ministerio de Salud & 277 & 60.6 \\
& El Ministerio de Educación & 80 & 17.5 \\
¿Cuál es la mejor solución & Eliminación de los canes (eutanasia) & 44 & 9.6 \\
para eliminar el problema & Esterilización de hembras & 361 & 79.0 \\
de los perros vagabundos? & Castración de machos & 278 & 60.8 \\
& Adopciones & 324 & 70.9 \\
& Otra & 49 & 10.7 \\
\hline
\end{tabular}

${ }^{1}$ Pregunta con opción de respuesta múltiple

da por el Código Sanitario para los Animales Terrestres de la OIE que indica que perro vagabundo es aquel animal que no esté bajo control directo de una persona o al que no se le impida errar libremente (OIE, 2010). En ese contexto, la percepción de los estudiantes no se ajustaría a la realidad, debido a que estudios realizados en diferentes lugares muestran lo contrario. Un ejemplo de ello es el estudio realizado en Brasil, donde en una muestra de 498 perros vagabundos, el 95\% tenía dueño (Ruiz de Matos et al., 2002). Por ello, se puede inferir que la presencia de perros vagabundos es consecuencia de una tenencia irresponsable (ICAM, 2007; Valencia,
2012). Si bien esta percepción es diferente según los años de estudio de los encuestados $(\mathrm{p}<0.05)$, no se encontró un patrón o tendencia (creciente o decreciente) en dicha percepción.

La percepción de que la desnutrición es el principal problema que sufren los perros vagabundos estaría asociado a los hallazgos de animales con baja condición corporal, como los encontrados en un estudio realizado en el distrito de Los Olivos, Lima, donde se observó que el 57-62\% de los perros vagabundos presentaban baja condición corporal (Ochoa et al., 2014). 
La percepción de los estudiantes sobre las parasitosis concuerda con estudios realizados en América Latina, incluso en Lima, donde se observa que la gran mayoría de los canes $(50-87 \%)$ presentan parasitosis intestinal (Iannacone et al., 2001; Llanos et al., 2010; Solarte-Paredes et al., 2013). Sin embargo, en cuanto a accidentes (54.9\%) y violencia física (49.0\%) sufrida por los perros, parece que excede a la realidad si se contrasta esta percepción con los resultados de un estudio en el distrito de Los Olivos, Lima, donde se reportó $27-29 \%$ de animales con lesiones en la piel y cojera (Ochoa et al., 2014), resultados que podrían representar una medida indirecta de la medición de dichas variables.

Los estudiantes perciben que la reproducción no deseada es el principal problema que ocasionan los perros vagabundos. Esto puede comprenderse si se considera que en países donde se ha desarrollado un adecuado control poblacional de canes a través de esterilización, el crecimiento poblacional de canes llega hasta un 8\% (Downes et al., 2009; Zumpano et al., 2011). La esterilización en ciudades de países en desarrollo que permita un control adecuado de la población de perros vagabundos es de difícil implementación, más aún si se considera la capacidad reproductiva de las perras (Sternheim, 2012).

El segundo problema a nivel de importancia percibido por los estudiantes es la transmisión de enfermedades de curso zoonótico. Aunque no se preguntó específicamente que enfermedades se transmitirían, es probable que estas involucren principalmente las parasitarias, entre ellas la toxocariasis. Esta es una enfermedad de importancia en salud pública, que se transmite a través de las larvas del parásito Toxocara spp, y en donde la principal fuente de infección para el humano son los parques contaminados con heces de perros (Chávez et al., 2002; Romero et al., 2009; Iannacone et al., 2012). Asimismo, se ha reportado que cerca del $50 \%$ de niños de educación primaria de una institución educativa estatal del distrito de San Juan de
Lurigancho, Lima, resultaron sero-positivos a toxocariasis (Breña et al., 2007).

La respuesta de los estudiantes acerca de que los perros vagabundos ocasionan accidentes por mordeduras tiene fundamento si se considera que aproximadamente la mitad de las mordeduras se producen en la calle (Ibarra et al., 2003). Un estudio realizado específicamente en población de niños encuentra que un tercio de los accidentes por mordeduras se produjeron en la vía pública (Morales et al., 2011). Por otro lado, la contaminación sonora producida por los perros no fue considerada por los estudiantes como un problema importante.

No se encontró concordancia en las respuestas sobre las instituciones que deberían enfrentar el problema de los perros vagabundos y de quiénes realmente lo hacen. La mayoría de los encuestados opinó que debería de enfrentarlo la municipalidad del distrito; no obstante, muy pocos refieren que esto se viene realizando, pese a que existe la Ley N. ${ }^{\circ} 27596$, Ley que Regula el Régimen Jurídico de Canes, donde se menciona que las municipalidades deben enfrentar este problema.

Un alto porcentaje de encuestados opinó que el problema de los perros vagabundos está siendo enfrentado por grupos proteccionistas y albergues. El Ministerio de Salud también fue percibido como una de las instituciones que debería enfrentar el problema de los perros vagabundos, probablemente porque entre sus funciones se encuentra la de velar por la tenencia de los perros en relación a las campañas de prevención de la rabia. Sin embargo, se debe de tener en cuenta que la tenencia no responsable de los animales de compañía es la que genera los problemas de perros vagabundos y que instituciones como municipalidades y el Ministerio de Salud solo enfrentan el problema una vez que se ha producido, a través de normas punitivas o de control poblacional en zonas de riesgo a rabia, respectivamente. 
La mayoría de estudiantes consideró que la esterilización de hembras sería la mejor solución para este problema; en contraste, un estudio menciona que para que esto funcione es necesario esterilizar al menos al $70 \%$ de la población (Sternheim, 2012). Ello, evidentemente, es difícil de conseguir, más aún en realidades como la de los países latinoamericanos, donde la preocupación por el control reproductivo es baja y la inversión en el tema es muy limitada.

La percepción acerca de que las adopciones representan una solución viable para el problema de los perros vagabundos se contrasta con los resultados de estudios en Europa, donde la población tiene mayores recursos económicos que la población latina, y la tasa de adopciones no supera el 10\% (Fatjó y Calvo, 2015). La eutanasia no fue mayormente considerada por los encuestados; no obstante, es una medida utilizada en varios países en situaciones de emergencia, como por ejemplo ante un brote de rabia urbana, tal cual sucedió en Indonesia (Windiyaningsih et al., 2004).

Los resultados del estudio muestran que la problemática de los perros vagabundos es percibida de diferente manera, lo que podría estar influenciado por la universidad de procedencia y el ciclo de estudio de los estudiantes, cuyo contraste no es parte del estudio. Sin embargo, se infiere la necesidad de que las currículas de todas las escuelas y facultades de medicina veterinaria incorporen la discusión de esta temática de manera uniforme y técnica, a fin de que los profesionales egresados de los centros de estudios compartan los mismos conocimientos y enfoques técnicos cuando tengan la necesidad de enfrentar este problema como parte de su actividad profesional.

\section{Conclusiones}

- La percepción acerca de si los perros vagabundos tienen dueño es baja $(36.5 \%)$.
- Los encuestados consideran que los principales problemas que sufren los perros vagabundos son la desnutrición, parasitosis y accidentes, y que los principales problemas que ocasionan son la reproducción no deseada, zoonosis y ruptura de bolsas de basura.

- Se les atribuye a la municipalidad del distrito y al Ministerio de Salud la responsabilidad de enfrentar este problema.

- Los estudiantes consideran como las opciones más viables para el control de perros vagabundos a la esterilización de hembras, adopciones y castración de machos.

\section{Literatura Citada}

1. Arauco D, Urbina B, León D, Falcón N. 2014. Indicadores demográficos y estimación de la población de canes con dueño en el distrito de San Martín de Porres, Lima-Perú. Salud Tecnol Vet 2: 83-92.

2. Breña J, Hernández R, Hernández A, Castañeda R, Espinoza Y, Roldán W, et al. 2011. Toxocariosis humana en el Perú: aspectos epidemiológicos, clínicos y de laboratorio. Acta Med Peruana 28: 228-236.

3. Breña J, Maguiña C, Rolando I, Hernández R, Hernández A. 2007. Evaluación clínica de 80 niños con infección por Toxocara canis. En: XVIII Congreso Federación Latinoamericana de Parasitología - FLAP.

4. Chávez A, Casas E, Cajas J, Velarde J. 2000. Contaminación de parques públicos con huevos de Toxocara spp en los distritos de la Provincia Constitucional del Callao y del Cono Sur de Lima Metropolitana. Rev Inv Vet Perú 11: 5257. doi: 10.15381/rivep.v11i1.6795

5. Chávez A, Casas E, Serrano M, Cajas J, Velarde J, La Rosa V, López J. 2002. Riesgo de contraer enfermedades parasitarias en los parques públicos de Lima y Callao. Rev Inv Vet Perú 13: 84-91. doi: 10.15381/rivep.v13i2.7337 
6. Downes M, Canty M, More S. 2009. Demography of the pet dog and cat population on the island of Ireland and human factors influencing pet ownership. Prev Vet Med 92:140-149. doi: 10.1016/ j.prevetmed.2009.07.005

7. Fatjó J, Calvo P. 2015. Estudio de la Fundación Affinity sobre el abandono, la pérdida y la adopción de animales de compañía en España 2015: interpretación de los resultados. Fundación Affinity Animales y Salud. [Internet]. Disponible en: http://www.fundacion-affinity.org/sites/ default/files/white-paper-estudiofundacion-affinity-abandono-adopcion2016-es.pdf

8. Garrido M, Zagalaz M, Torres G, Romero $S$. 2010. Diseño y validación de un cuestionario para técnicos deportivos acerca de su opinión sobre las actitudes de padres y madres en el deporte (CTPMD). Cuadernos Psicol Deporte 10(2): 7-21.

9. Iannacone J, Cordova K, Wong $R$. 2001. Estructura comunitaria de helmintos de perros vagabundos de San Juan de Lurigancho, Lima, Perú. Rev Bras Zool 18: 277-288. doi: 10.1590/ S0101-81752001000500024

10. Iannacone J, Alvariño L, CárdenasCallirgos J. 2012. Contaminación de los suelos con huevos de Toxocara canis en parques públicos de Santiago de Surco, Lima, Perú. Neotrop Helminthol 6: 97-108.

11. Ibarra L, Morales M, Cáceres L. 2003. Mordeduras a personas por ataque de perros en la ciudad de Santiago, Chile. Avances Cienc Vet 18: 41-46.

12. [ICAM] Coalición Internacional para el Manejo de Animales de Compañía. 2007. Guía para el manejo humanitario de poblaciones caninas. 24 p. [Internet]. Disponible en: http://www.icamcoalition.org/downloads/Guia_Para El Manejo Humanitario de Poblaciones Caninas_Spanish.pdf

13. La Rosa V, Chávez A, Casas E. 2001. Contaminación de parques públicos del cono norte con huevos de Toxocara spp.
Rev Inv Vet Perú 12: 116-121. doi: 10.15381/rivep.v12i1.7431

14. López F, Chávez A, Casas, E. 2005. Contaminación de los parques públicos de los distritos de Lima Oeste con huevos de Toxocara sp. Rev Inv Vet Perú 16:77-81. doi: 10.15381/rivep.v16i1.1543

15. Llanos M, Condori M, Ibañez T, Loza M. 2010. Parasitosis entérica en caninos (Canis familiaris) en el área urbana de Coroico, Nor Yungas, Departamento de La Paz, Bolivia. J Selva Andina Res Soc 1(1): 37-49.

16. Molina JL, Faigenbaum A, Castro JR, Gastrel H, Ruggia R. 2006. Estimación de la población de caninos vagabundos en la Ciudad de Buenos Aires, octubre-noviembre 2006. Rev Argentina Zoonosis Enf Infec Emerg 4(4):136-139.

17. Morales C, Falcón N, Hernández H, Fernández C. 2011. Accidentes por mordedura canina, casos registrados en un hospital de niños de Lima, Perú 19952009. Rev Perú Med Exp Salud Pública 28: 639-642.

18. Ochoa Y, Falcón N, Zuazo R, Guevara P. 2014. Estimación de la población de perros callejeros en el distrito de Los Olivos, Lima, Perú. Rev Inv Vet Perú 25: 366373. doi: 10.15381/rivep.v25i3.-10114

19. [OIE] Organización Mundial para la Salud Animal. 2010. Código Sanitario para los Animales Terrestres. Cap 7.7. El control de las poblaciones de perros vagabundos. [Internet]. Disponible en: http://web.oie.int/esp/normes/mcode/ es_chapitre_11.7.7.htm

20. Paredes A, Zuazo J. 2014. Características de los ingresantes a la carrera de Medicina Veterinaria y Zootecnia de la Universidad Peruana Cayetano Heredia en el periodo 2010-2013. Salud Tecnol Vet 2: 113-119. doi: 10.20453/ stv.v2i2.2248

21. Ramírez J, Falcón N, Serrano E. 2014. Contaminación de suelos con huevos de Toxocara sp en ambientes internos de instituciones educativas estatales de los distritos del cono norte de Lima. Salud Tecnol Vet 2: 78-82. 
22. Romero C, García A, Mendoza G, Torres $N$, Ramírez N. 2009. Contaminación por Toxocara spp en parques de Tulyehualco, México. Rev Cient FCVLUZ 19: 253-256.

23. Romero C, Hernández H, Falcón N. 2013. Costos de hospitalización de los accidentes por mordedura de canes en un hospital de niños en Lima-Perú, periodo 2006-2010. Salud Tecnol Vet 1:2632. doi: 10.20453/stv.v1i2.109

24. Ruiz de Matos M, Porto M, Reichmann M, Dominguez M. 2002. Sao Paulo Pasteur Institute Technique for estimating a canine population. Cade Saúde Pública 18: 1423-1428 [in Portuguese].

25. Salamanca CA, Polo LJ, Vargas J. 2011. Sobrepoblación canina y felina: tendencias y nuevas perspectivas. Rev Med Vet Zoot 58(1): 45-53.

26. Sánchez E. 2007. Curso de introducción a la investigación clínica. Cap 6: Diseño de cuestionarios. SEMERGEN 33: 412-416.

27. Schvartzman S, Pacín M. 2005. Lesiones por mordedura de perro en niños. Arch Argent Pediatr 103: 389-395.

28. Seoane T, Martín JL, Martín-Sánchez E, Lurueña-Segovia S, Alonso FJ. 2007. Curso de introducción a la investi- gación clínica. Capítulo 7: estadística: estadística descriptiva y estadística inferencial. SEMERGEN 33: 466-471.

29. Solarte-Paredes L, CastañedaSalazar R, Pulido-Villamarín A. 2013. Parásitos gastrointestinales en perros callejeros del Centro de Zoonosis de Bogotá DC, Colombia. Neotrop Helminthol 7: 83-93.

30. Sternheim I. 2012. Perros callejeros en los países bajos. DogResearch. [Internet] Disponible en: http:// www.doctordog.n1/DR_Espanol_Perros Callejeros[1].pdf

31. Valencia C. 2012. Técnicas de control de poblaciones caninas callejeras usadas a nivel mundial. Tesis de Médico Veterinario. Valdivia: Universidad Austral de Chile. $26 \mathrm{p}$.

32. Windiyaningsih $\mathrm{C}$, Wilde $\mathrm{H}$, Meslin $\mathrm{F}$, Suroso T, Widarso H. 2004. The rabies epidemic on Flores Island, Indonesia. J Med Assoc Thai 87(11):1389-1393.

33. Zumpano R, Tortosa A, Degregorio O. 2011. Estimación del impacto de esterilización en el índice de crecimiento de la población de caninos. Rev Inv Vet Perú 22: 336-341. doi: 10.15381/rivep. v22i4.333 